\title{
Osteopathic Potential of Methotrexate: Medial Tibial Stress Syndrome
}

\author{
Canan CİMŞIT, Onur BUĞDAYCI, Mustafa Erkin ARIBAL \\ Department of Radiology, Marmara University Pendik Training and Research Hospital, İstanbul, Turkey
}

Methotrexate (MTX) is a folic acid antagonist commonly used for the treatment of malignancies, autoimmune diseases, and chronic inflammatory conditions like rheumatoid arthritis and psoriatic arthritis. ${ }^{1,2}$ Methotrexate osteopathy is one of the side effects of the drug when used in high doses and is characterized by bone pain, osteopenia, and insufficiency fractures. ${ }^{1}$ This spectrum of MTX osteopathy was previously studied in patients on low-to intermediate doses and the results varied.1,3-5 Shin splints or medial tibial stress syndrome (MTSS) is caused in response to the chronic repetitive stress on the posteromedial border of the tibia and seen mostly in athletes and military personnel. ${ }^{6}$ To our knowledge, there are only a few reports in literature describing MTSS in patients with rheumatoid arthritis and psoriatic arthritis under MTX treatment. ${ }^{3,6,7}$ In this article, we report a 59-year-old female patient with a three-year history of rheumatoid arthritis who presented with severe pain and swelling of the tibia. She described the pain on the anterior and medial aspect of the lower leg not relieved by rest. She had no predisposing history of physical activity. She was under MTX (10 mg/week) and low dose prednisolone therapy. On laboratory analysis, C-reactive protein value was $0.20 \mathrm{mg} / \mathrm{dL}$, sedimentation rate was $29 \mathrm{~mm} /$ hour, and dual energy X-ray absorptiometry showed osteopenia. Extremity X-ray was normal. Magnetic resonance imaging (MRI) revealed periosteal edema as hyperintensity on fat-saturated $\mathrm{T}_{2}$-weighted images, adjacent to the outer surface of the medial cortex of mid-to-distal tibial diaphysis (Figure 1). Regression of the patient's complaints in one month after lowering the dosage of MTX endorsed the radiological diagnosis.

The clinical diagnosis of MTSS was defined according to Yates and White 8 as having exercise induced pain on the posterolateral side of the tibia, and pain on palpation along a length of at least $5 \mathrm{~cm}$ over this area. History of tibial fracture and clinical suspicion of compartment syndrome were the exclusion criteria.
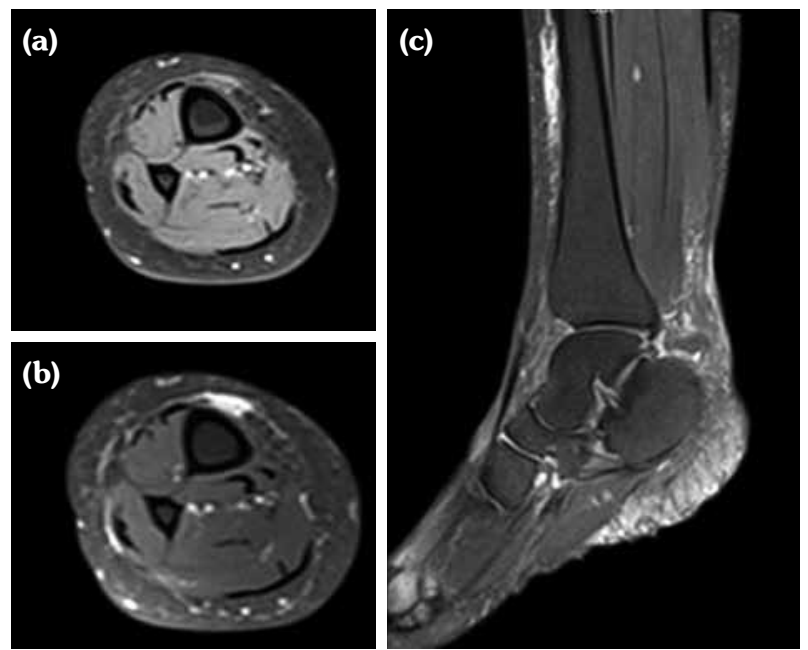

Figure 1. Magnetic resonance imaging revealed no signal change adjacent to outer surface of medial cortex of tibia on fat-saturated $\mathrm{T}_{1}$-weighted axial image (a). Note juxtaosseous band-like high signal fixed to periosteum on fatsaturated $\mathrm{T}_{2}$-weighted images $(\mathbf{b}, \mathbf{c})$. 
Magnetic resonance imaging is more sensitive than radiography since X-ray is often normal in early stages. ${ }^{9}$ Fredericson $^{10}$ developed MRI classification for tibial stress injuries and there are new modified classifications in search of the comparison of MRI classification with severity and clinical outcome. According to the classification, grade 1 represents periosteal edema only. The periosteal edema was considered mild if it involved less than $25 \%$ and severe if it involved more than $50 \%$ of the circumference of the tibial cortex. Grade 2 is defined by bone marrow edema visible on $\mathrm{T}_{2}$-weighted images whereas in grade 3 , bone marrow edema is seen both on $\mathrm{T}_{1}$-weighted and $\mathrm{T}_{2}$-weighted images. Grade 4 injury represents intracortical focal or linear signal abnormalities. Our patient's MRI findings revealed grade 1 mild tibial stress injury.

Methotrexate osteopathy is a rare complication during long-term low dose MTX treatment where the distal tibia is most commonly affected. MTSS should be included to the clinical spectrum of MTX osteopathy. Not only orthopedic surgeons and physiatrists but also rheumatologists and oncologists should be aware of MTSS in patients on MTX treatment complaining of persistent leg pain. MRI should be performed for early diagnosis since MTSS can progress to insufficiency fracture in inflammatory arthritis patients if untreated.

\section{Declaration of conflicting interests}

The authors declared no conflicts of interest with respect to the authorship and/or publication of this article.

\section{Funding}

The authors received no financial support for the research and/or authorship of this article.

\section{REFERENCES}

1. Meier L, van Tuyll van Sersooskerken AM, Liberton E, Kleijn L, Westgeest T, Polak M, et al. Fractures of the proximal tibia associated with longterm use of methotrexate: 3 case reports and a review of literature. J Rheumatol 2010;37:2434-8.

2. Torikai E, Kageyama Y, Takahashi M, Nagano A. The effect of methotrexate on bone metabolism markers in patients with rheumatoid arthritis. Mod Rheumatol 2006;16:350-4.

3. Alonso-Bartolomé P, Martinez-Taboada VM, Canga $A$, Blanco R. Medial tibial stress syndrome due to methotrexate osteopathy. Ann Rheum Dis 2006;65:832-3.

4. Rozin AP. Is methotrexate osteopathy a form of bone idiosyncrasy? Ann Rheum Dis 2003;62:1123.

5. Minaur NJ, Jefferiss C, Bhalla AK, Beresford JN. Methotrexate in the treatment of rheumatoid arthritis. In vitro effects on cells of the osteoblast lineage. Rheumatology 2002;41:735-40.

6. Lee JH, Kwok SK, Park SH, Kim HY, Park KS. Medial tibial stress syndrome progressing to tibial fracture in rheumatoid arthritis. Int $J$ Rheum Dis 2014;17:125-6.

7. Moen M, Weir A, Rijthoven A, Reurink G, Tol JL, Backx F. Medial tibial stress syndrome induced by methotrexate: a case report. Turk $\mathrm{J}$ Rheumatol 2011;26:258-61.

8. Yates $\mathrm{B}$, White $\mathrm{S}$. The incidence and risk factors in the development of medial tibial stress syndrome among naval recruits. Am J Sports Med 2004;32:772-80.

9. Kijowski R, Choi J, Shinki K, Del Rio AM, De Smet A. Validation of MRI classification system for tibial stress injuries. AJR Am J Roentgenol 2012;198:878-84.

10. Fredericson M, Bergman AG, Hoffman KL, Dillingham MS. Tibial stress reaction in runners: correlation of clinical symptoms and scintigraphywith a new magnetic resonance imaging grading system. Am J Sports Med 1995;23:472-81. 